\title{
Gonadal steroid-dependent effects on bone turnover and bone mineral density in men
}

Joel S. Finkelstein, ${ }^{1}$ Hang Lee, ${ }^{2}$ Benjamin Z. Leder, ${ }^{1}$ Sherri-Ann M. Burnett-Bowie, ${ }^{1}$ David W. Goldstein, ${ }^{1}$ Christopher W. Hahn, ${ }^{1}$ Sarah C. Hirsch, ${ }^{1}$ Alex Linker, ${ }^{1}$ Nicholas Perros, ${ }^{1}$ Andrew B. Servais, ${ }^{1}$ Alexander P. Taylor, ${ }^{1}$ Matthew L. Webb, ${ }^{1}$ Jonathan M. Youngner, ${ }^{1}$ and Elaine W. Yu' ${ }^{1}$

'Endocrine Unit, Department of Medicine and 'Biostatistics Center, Massachusetts General Hospital, Boston, Massachusetts, USA.

\begin{abstract}
BACKGROUND. Severe gonadal steroid deficiency induces bone loss in adult men; however, the specific roles of androgen and estrogen deficiency in hypogonadal bone loss are unclear. Additionally, the threshold levels of testosterone and estradiol that initiate bone loss are uncertain.
\end{abstract}

\begin{abstract}
METHODS. One hundred ninety-eight healthy men, ages 20-50, received goserelin acetate, which suppresses endogenous gonadal steroid production, and were randomized to treatment with $0,1.25,2.5,5$, or 10 grams of testosterone gel daily for 16 weeks. An additional cohort of 202 men was randomized to receive these treatments plus anastrozole, which suppresses conversion of androgens to estrogens. Thirty-seven men served as controls and received placebos for goserelin and testosterone. Changes in bone turnover markers, bone mineral density (BMD) by dual-energy x-ray absorptiometry (DXA), and BMD by quantitative computed tomography (QCT) were assessed in all men. Bone microarchitecture was assessed in 100 men.

RESULTS. As testosterone dosage decreased, the percent change in C-telopeptide increased. These increases were considerably greater when aromatization of testosterone to estradiol was also suppressed, suggesting effects of both testosterone and estradiol deficiency. Decreases in DXA BMD were observed when aromatization was suppressed but were modest in most groups. QCT spine BMD fell substantially in all testosterone-dose groups in which aromatization was also suppressed, and this decline was independent of testosterone dose. Estradiol deficiency disrupted cortical microarchitecture at peripheral sites. Estradiol levels above $10 \mathrm{pg} / \mathrm{ml}$ and testosterone levels above $200 \mathrm{ng} / \mathrm{dl}$ were generally sufficient to prevent increases in bone resorption and decreases in BMD in men.
\end{abstract}

CONCLUSIONS. Estrogens primarily regulate bone homeostasis in adult men, and testosterone and estradiol levels must decline substantially to impact the skeleton.

TRIAL REGISTRATION. ClinicalTrials.gov, NCT00114114.

FUNDING. AbbVie Inc., AstraZeneca Pharmaceuticals LP, NIH.

\section{Introduction}

Gonadal steroids have powerful effects on bone. During puberty, increases in gonadal steroids stimulate osteoblast activity, causing bone mineral density (BMD) to increase markedly (1). At midlife in women, gonadal steroids decline to prepubertal levels, which in turn increases bone resorption and leads to rapid bone loss (2). Unlike in women, decreases in gonadal steroids in middle-aged and elderly men are quite modest $(3,4)$. If adult men develop severe hypogonadism, however, as happens in men receiving gonadotropin-releasing hormone $(\mathrm{GnRH})$ agonist therapy for prostate cancer, bone resorption increases and leads to rapid bone loss $(5,6)$. The levels to which gonadal steroids must be reduced

Role of the funding source: The sponsors of the study had no role in study design, data collection, data analysis, data interpretation, or drafting of the manuscript. Sponsors were allowed to review the manuscript to ensure proper acknowledgement of their support. Conflict of interest: The authors have declared that no conflict of interest exists. Submitted: August 14, 2015; Accepted: December 10, 2015

Reference information: / Clin Invest. 2016;126(3):1114-1125. doi:10.1172/JCI84137. to initiate bone loss in men are unknown, however. This issue is clinically important because male aging is associated with modest declines in both gonadal steroids and BMD (7), but it is not known whether those changes are causally related. Confirmation of causality and identification of thresholds below which skeletal effects are apparent would help to guide evidence-based decisions about testosterone replacement therapy in adult men.

Over $80 \%$ of circulating estradiol in men is derived from aromatization of testosterone (8). Thus, serum testosterone and estradiol levels are correlated (9), and the prototypical changes in hypogonadal men - including alterations in body composition, decreases in sexual function, and high turnover bone loss - could be due to androgen deficiency, estrogen deficiency, or both (10). Several findings have been cited as evidence supporting a pivotal role of estrogen in adult male skeletal homeostasis (11). However, they do not definitively establish a causal role for estrogen deficiency in adult male hypogonadal bone loss. In this study, we utilized a variety of pharmacologic interventions to isolate the specific roles of androgens and estrogens in the pathogenesis of hypogonadal bone loss in 

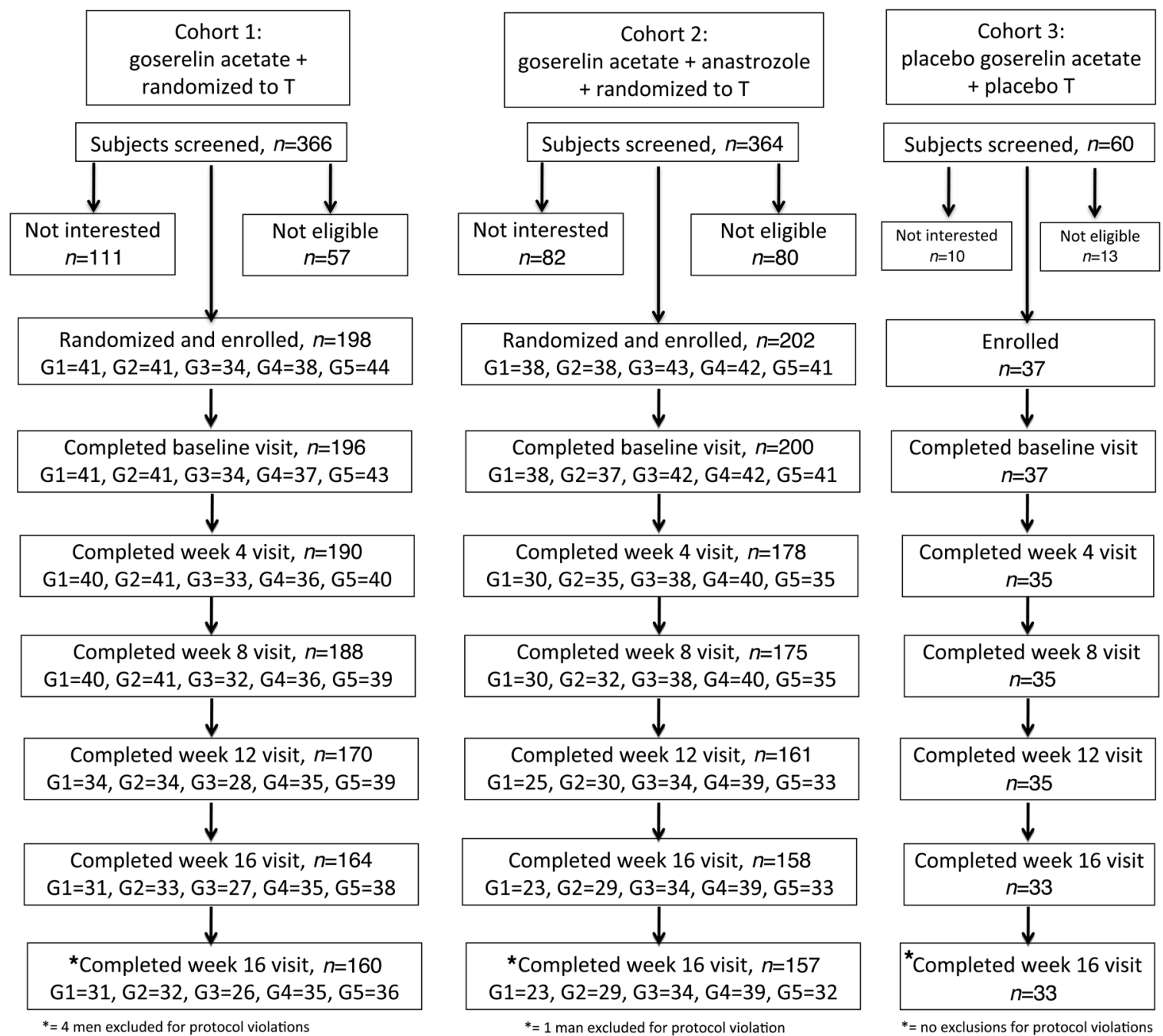

Figure 1. Trial profile. T, testosterone gel. G, group. G1, $0 \mathrm{~g}$ (placebo) testosterone gel daily; $\mathrm{G} 2,1.25 \mathrm{~g}$ of testosterone daily; $\mathrm{G} 3,2.5 \mathrm{~g}$ of testosterone daily; $\mathrm{C} 4,5 \mathrm{~g}$ of testosterone gel daily; $\mathrm{G} 5,10 \mathrm{~g}$ of testosterone gel daily.

adult men and to determine the levels of testosterone and estradiol at which the risk of hypogonadal bone loss begins to increase.

\section{Results}

Baseline characteristics. Figure 1 shows details related to recruitment into each of the three cohorts, the randomization of subjects into each of the five testosterone-dose groups in cohorts 1 and 2, and the completion rates for each testosterone-dose group at each study visit. Table 1 shows the baseline characteristics of the three cohorts and Supplemental Table 1 (supplemental material available online with this article; doi:10.1172/JCI84137DS1) shows the self-reported race/ethnicity of the men in each cohort. There were no significant differences in baseline characteristics between the controls and any of the groups in cohorts 1 or 2. Other than a slightly higher mean baseline serum testosterone level in the men who dropped out early in cohort 1 ( $513 \pm 135$ vs. $569 \pm 179 \mathrm{ng} / \mathrm{dl}, P$ $<0.05)$, there were no significant differences in baseline characteristics between the men who completed the protocol and those who discontinued participation before week 16 in any cohort. Reasons for early discontinuation are summarized in Supplemental Table 2.

Hormone levels. Figure 2 shows the mean serum testosterone and estradiol levels in relation to testosterone dose in each study group in cohort 1 (blue dots), cohort 2 (red dots), and the controls (black dots). Mean serum testosterone levels on therapy ranged from $44 \pm 13$ to $805 \pm 355 \mathrm{ng} / \mathrm{dl}$ in cohort 1 and from $41 \pm 13$ to $924 \pm 521 \mathrm{ng} / \mathrm{dl}$ in cohort 2 . Mean serum estradiol levels ranged from $3.6 \pm 1.4$ to $33 \pm 15 \mathrm{pg} / \mathrm{ml}$ in cohort 1 and from $1.0 \pm 0.4$ to $2.8 \pm 1.8 \mathrm{pg} / \mathrm{ml}$ in cohort 2 . Mean serum testosterone and estradiol levels were $591 \pm 173 \mathrm{ng} / \mathrm{dl}$ and $29 \pm 9 \mathrm{pg} / \mathrm{ml}$ in the controls. Serum testosterone and serum estradiol levels were highly correlated and best fit by a linear regression $\left(\mathrm{E}_{2}=0.0356(\mathrm{~T})+4.4003 ; \mathrm{R}=0.79\right)$.

Effects oftestosterone dose and level on bone turnover markers. Figure 3 shows the percent change in serum C-terminal telopeptide of type 1 collagen (CTX) and procollagen type- 1 amino-terminal propeptide (P1NP) in relation both to testosterone dose and to the mean serum testosterone levels during weeks $4-16$ in men who received testosterone with (cohort 2) and without (cohort 1) coadministration of anastrozole. Within cohort 1 (blue dots), serum CTX levels increased 


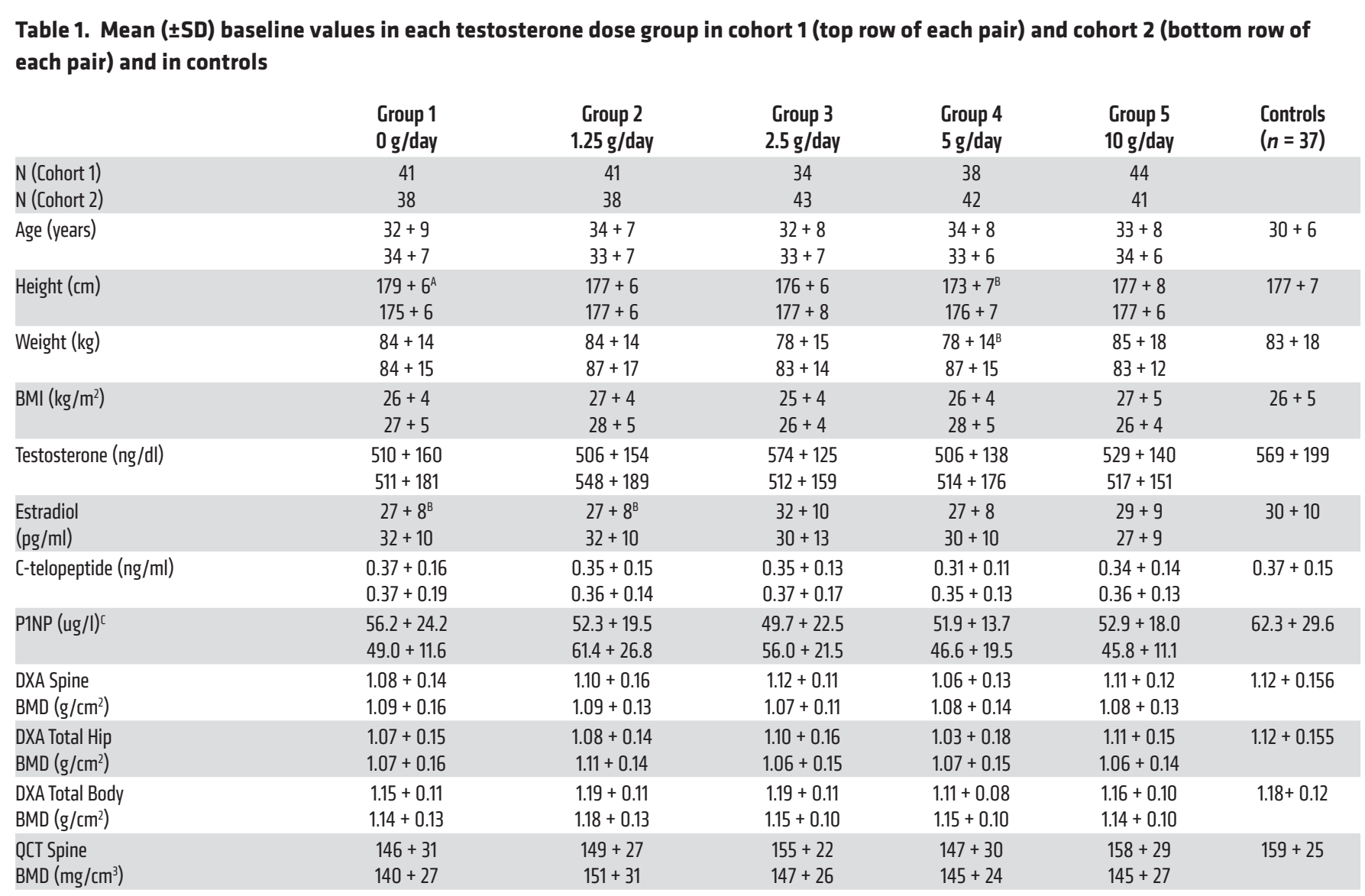

To convert testosterone to $\mathrm{nmol} / \mathrm{l}$, multiply by 0.03467 . To convert estradiol to $\mathrm{pmol} / \mathrm{I}$, multiply by $3.671 .{ }^{A} P<0.01$ using independent $t$ test to compare baseline values of cohort 1 and cohort 2 for groups assigned to the same testosterone dose. ${ }^{\mathrm{B}} P<0.05$ using independent $t$ test to compare baseline values of cohort 1 and cohort 2 for groups assigned to the same testosterone dose. ${ }^{C} P<0.05$ with the use of one-way ANOVA for comparisons across dose groups in cohort 2 .

significantly more than in the controls (black dots) only in men who received goserelin plus 0 (placebo) or 1.25 grams of testosterone daily or in men whose mean testosterone levels were below $200 \mathrm{ng} / \mathrm{dl}$. Serum P1NP only increased significantly in men treated with placebo or whose mean serum testosterone level was below $100 \mathrm{ng} / \mathrm{dl}$.

Within cohort 2 (red dots), serum CTX levels increased significantly more than in the controls in every testosterone group $(P<0.05$ for each comparison), with increases exceeding those observed in cohort 1 by $50 \%-100 \%$. Serum CTX even increased substantially more in cohort 2 than in cohort 1 in men who received placebo testosterone (Figure 3A), possibly because serum estradiol levels were higher in men who received placebo testosterone alone (cohort 1 , $3.6 \pm 1.4 \mathrm{pg} / \mathrm{ml}$ ) than in men who received placebo testosterone plus anastrozole (cohort 2, $1.0 \pm 0.4 \mathrm{pg} / \mathrm{ml}$ ). Within cohort 2, there was a significant inverse relationship between the testosterone dose and the increase in serum CTX levels. CTX levels increased more in the groups that received $0,1.25$, or 2.5 grams of testosterone gel daily than in the 2 higher-dose groups, and these differences persisted even when the results were adjusted for the small differences in serum estradiol levels between testosterone-dose groups in cohort 2. Serum P1NP tended to increase more in groups that received anastrozole than in those that did not, though most of the individual comparisons were not statistically significant.
Effects of testosterone dose and level on BMD. Within cohort 1 , areal BMD of the lumbar spine, total hip, and total body by dual-energy x-ray absorptiometry (DXA) tended to decline as the dose or level of testosterone declined, though the magnitude of the changes in BMD was small and no change was significantly different from the controls (Figure 4, A-F, blue dots). Changes in trabecular spine BMD, assessed by quantitative computed tomography (QCT), were greater than changes in DXA BMD. Specifically, trabecular spine bone loss was detectable (3.0\%-5.8\% within 16 weeks) in the three groups that received the lowest testosterone doses or that had the lowest testosterone levels on therapy, although only the changes in the group that received 1.25 grams of testosterone daily and the group whose mean testosterone level on therapy was between 100 and $199 \mathrm{ng} / \mathrm{dl}$ were significantly different from the controls (Figure 4, G and $\mathrm{H}$, blue dots).

Within cohort 2, BMD by DXA declined by approximately $1 \%-2 \%$ in all dose groups at all skeletal sites; for each site, the decline in BMD appeared to be independent of testosterone dose or level (Figure 4, red dots). There were a few individual group comparisons that were significantly different from the controls, though these differences did not follow any predictable pattern. Trabecular spine BMD by QCT declined by approximately $4 \%-5 \%$ in each group in cohort 2 . These decreases were significantly 

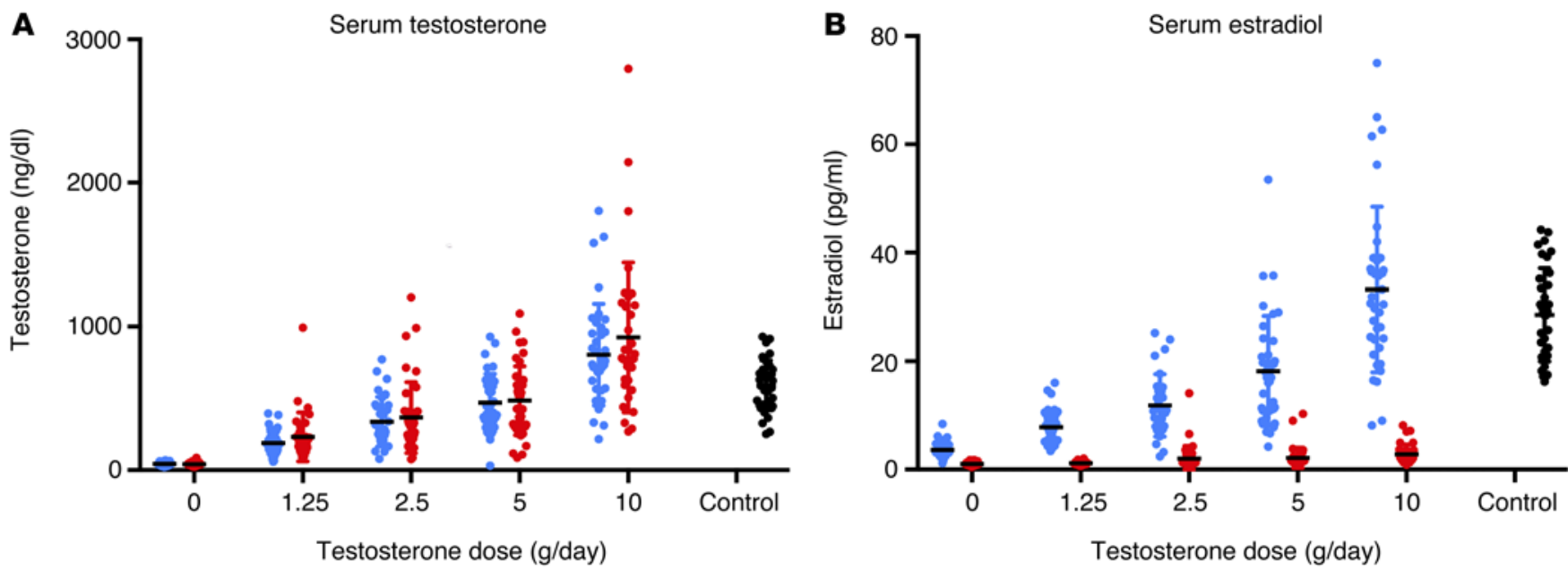

Figure 2. Serum testosterone and estradiol levels on treatment according to testosterone dose in individual subjects. (A and B) Subjects in cohort 1 (blue dots, $n=184$ ) received goserelin acetate plus 0 (placebo), 1.25, 2.5, 5, or $10 \mathrm{~g}$ of testosterone gel daily without anastrozole. Subjects in cohort 2 (red dots, $n=174$ ) received the same treatments plus anastrozole $1 \mathrm{mg}$ per day. Cohort 3 (controls, black dots, $n=35$ ) received placebos for both goserelin acetate and for the testosterone gel. The horizontal black line represents the mean, and the error bars represent $\pm 1 \mathrm{SD}$.

greater than in the controls for each testosterone-dose group and at each testosterone level, except for the group whose mean levels ranged from 100-199 ng/dl (Figure 4, G and H, red dots).

Relationship of estradiol to skeletal outcomes. Figure 5 shows the changes in bone turnover and BMD in cohort 1 in relation to mean estradiol levels on therapy. Serum CTX levels were stable until serum estradiol levels fell to $5-9.9 \mathrm{pg} / \mathrm{ml}$, at which point increases in serum CTX were significantly greater than in the controls $(P<$ 0.05). There was a further significant increase in serum CTX levels in men whose estradiol levels were below $5.0 \mathrm{pg} / \mathrm{ml}(P<0.05$ vs. men with estradiol levels of 5.0-9.9 pg/ml). Serum P1NP levels increased more than in controls in men whose estradiol levels were below $5 \mathrm{pg} / \mathrm{ml}$. There was an unexpected increase in P1NP levels in men whose estradiol levels were greater than $35 \mathrm{pg} / \mathrm{ml}$. Total hip and total body BMD by DXA and trabecular spine BMD by QCT all appeared to decline in men whose estradiol levels were 10.0-14.9 $\mathrm{pg} / \mathrm{ml}$, though most of these changes were not significantly different from the controls, whereas spine BMD by DXA appeared to decline in men whose estradiol levels were less than $10 \mathrm{pg} / \mathrm{ml}$.

A potential effect of estradiol on each outcome measure was also assessed by testing for a significant cohort-testosterone dose interaction between cohorts 1 and 2 and by comparing the mean change in each outcome measure between the groups that received any active dosage of testosterone (i.e., groups $2,3,4$, and 5) in cohorts 1 and 2. The cohort-testosterone dose interaction was significant for lumbar spine BMD by DXA $(P=0.0027)$ and borderline significant for total body BMD $(P=0.089)$ and trabecular BMD by QCT $(P=0.083)$, suggesting an independent effect of estradiol on these measures. Serum CTX levels increased more and BMD decreased more at all skeletal sites in men who received active testosterone gel (groups 2, 3, 4, and 5) plus anastrozole than in men who received active testosterone gel without aromatase inhibition ( $P=0.0207$ for total hip BMD, $P<0.0001$ for all other measures).

Assessment of the contributions of androgens versus estrogens to the regulation of peripheral volumetric BMD and bone microarchitecture. Table 2 shows the changes in volumetric BMD (vBMD) and bone microarchitecture by high resolution peripheral quantitative computed tomography (HR-pQCT) at the distal radius and distal tibia in a subset of men in cohort 2. Changes in vBMD at both the radius and the tibia were similar across testosterone-dose groups and were independent of testosterone dose, suggesting that testosterone does not affect VBMD in the setting of estrogen deficiency. However, total vBMD at the tibia declined from baseline within most testosterone-dose groups (Supplemental Figure 1), suggesting an independent effect of estradiol. Decreases in both cortical and trabecular vBMD contributed to the decline in total vBMD at both the radius and the tibia in men in whom aromatization of testosterone to estradiol was suppressed.

There were no significant differences between testosteronedose groups in the changes of indices of skeletal microarchitecture at either the radius or the tibia (Table 2 and Supplemental Figure 2), again suggesting a lack of testosterone effect in the setting of low estrogen. Furthermore, changes in indices of microarchitecture were not related to the dosage of testosterone, though there was a nonsignificant trend toward an increase in cortical porosity as the dose of testosterone was lowered. Cortical area decreased and trabecular area increased at both the radius and the tibia when aromatization of testosterone to estradiol was inhibited, suggesting that estrogen deficiency promotes endosteal resorption. There was also a tendency for cortical thickness to decline with estrogen deficiency, particularly at the tibia. There were no significant changes in trabecular number or trabecular thickness of the radius or the tibia, either in any individual testosterone-dose group or with pooled group analyses.

\section{Discussion}

In this study, we examined the roles of androgens and estrogens on bone turnover; BMD by DXA, QCT, and HR-pQCT; and bone microarchitecture using HR-pQCT in adult men receiving a GnRH agonist and a series of doses of testosterone, with or without a potent aromatase inhibitor. Our goals were (i) to determine whether bone metabolism is primarily regulated by androgens or 
By $T$ dose group
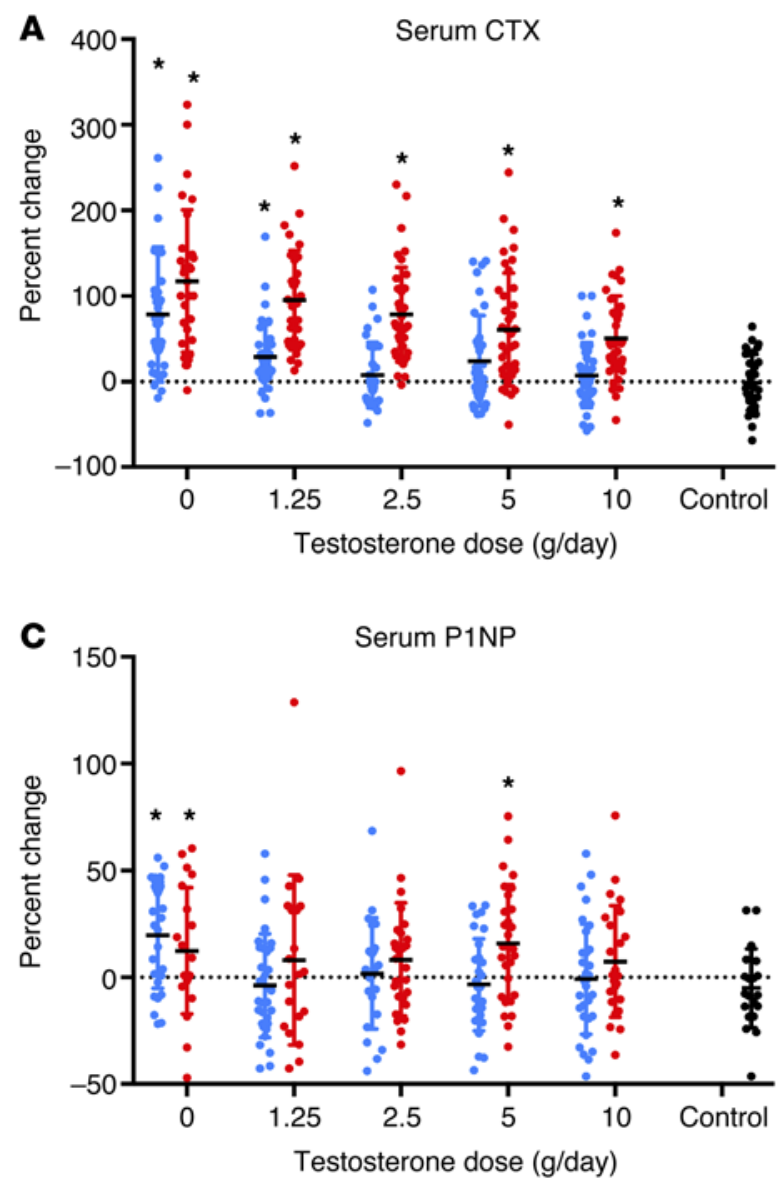

By serum T levels
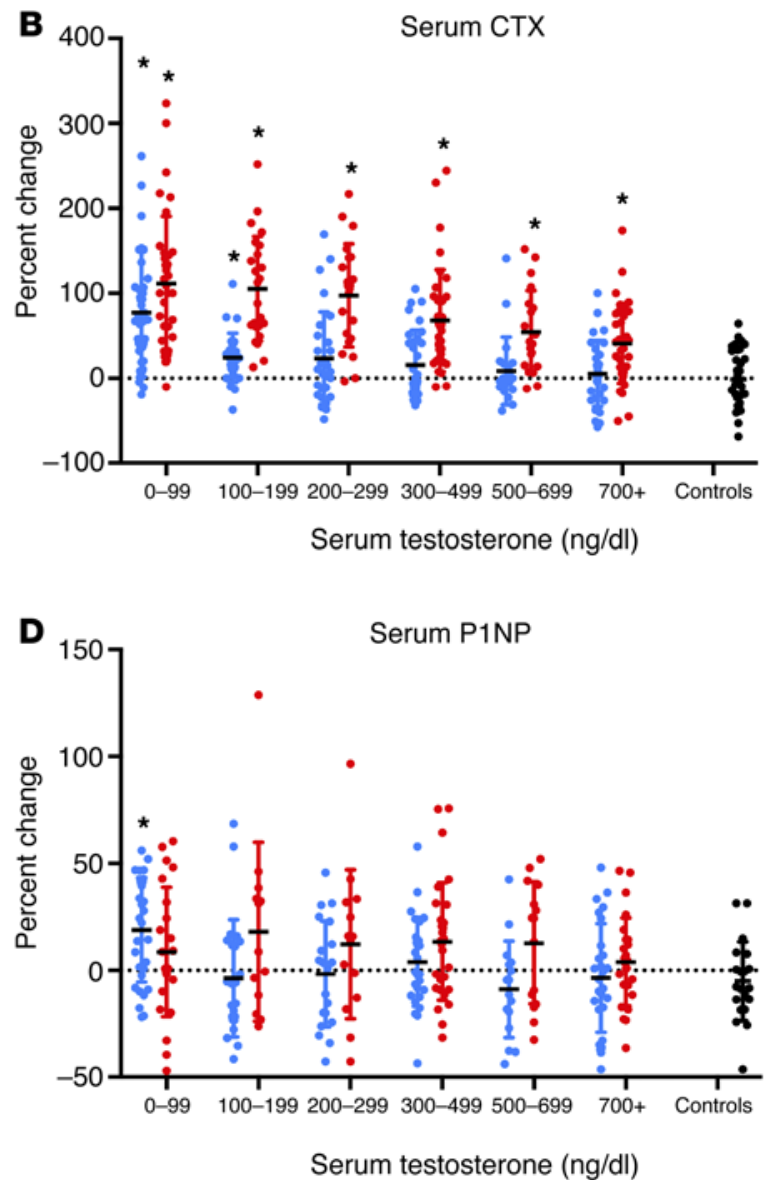

Figure 3. Percent change from baseline in bone turnover markers according to testosterone dose and mean serum testosterone levels on treatment in individual subjects. (A-D) Results are shown for CTX (A and B) and P1NP (C and D). Subjects in cohort 1 (blue dots, $n=184$ ) received testosterone gel daily without anastrozole. Subjects in cohort 2 (red dots, $n=174$ ) received the same treatments plus anastrozole 1 mg per day. Subjects in cohort 3 (controls, black dots, $n=35$ ) received placebos for both goserelin acetate and for the testosterone gel. The horizontal black line represents the mean, and the error bars represent $\pm 1 \mathrm{SD}$. $\mathrm{T}$, testosterone. ${ }^{*} P<0.05$ using Duncan's multiple range test.

estrogens in adult men and (ii) to determine the approximate levels of circulating testosterone or estradiol at which bone turnover increases and/or BMD or bone microarchitecture decreases.

Results from several types of studies suggest that estradiol plays a significant role in regulating bone metabolism in adult men. First, in both cross-sectional and longitudinal observational studies, associations between BMD and/or fracture risk and circulating estradiol levels are stronger than their associations are with circulating testosterone levels. The associations are all quite weak, however, generally explaining no more than a few percent of the variation in the data $(9,12)$. Moreover, because more than $80 \%$ of circulating estradiol in men is derived from aromatization of testosterone (8), serum testosterone and estradiol levels are significantly associated with each other, making it difficult to separate their individual contributions in observational studies.

Case reports of osteoporosis in genetic males with rare but illustrative null mutations of the estrogen receptor (ER) or androgen receptor (AR) genes $(13,14)$, or of the aromatase gene $(15,16)$, are frequently cited as evidence that both androgens and estro- gens play physiologically important roles in bone metabolism. However, because these subjects have congenital disorders, it is likely that their skeletal phenotypes reflect effects of gonadal steroids during bone development rather than effects of gonadal steroids on the adult male skeleton.

Studies utilizing medications as physiologic probes to produce a state of selective, reversible estrogen deficiency provide the most compelling evidence that estrogen deficiency, rather than androgen deficiency, is responsible for most of the skeletal manifestations in men with adult-onset hypogonadism. For example, we and others have reported that GnRH agonist-induced suppression of gonadal steroids, coupled with administration of testosterone and a potent aromatase inhibitor (to restore serum testosterone levels to the mid-portion of the reference range while estradiol levels remain low), increases bone resorption, though not to the extent observed in men treated with a GnRH agonist alone (17-19). One study in which an aromatase inhibitor was administered to men without GnRH agonist blockade reported a small decrease in spine BMD by DXA, although there were no significant changes in DXA BMD of the hip 


\section{By T dose group}
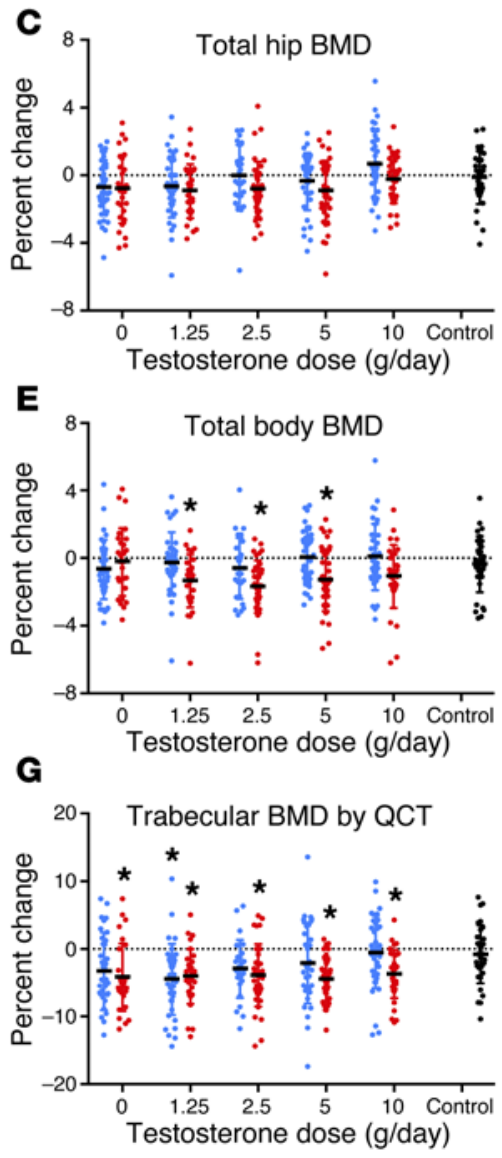
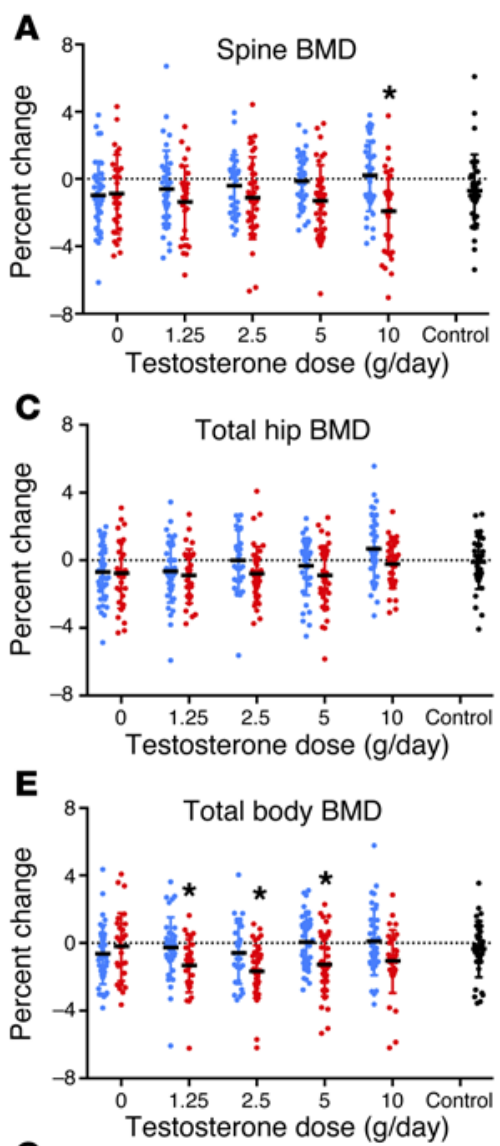

By serum T levels
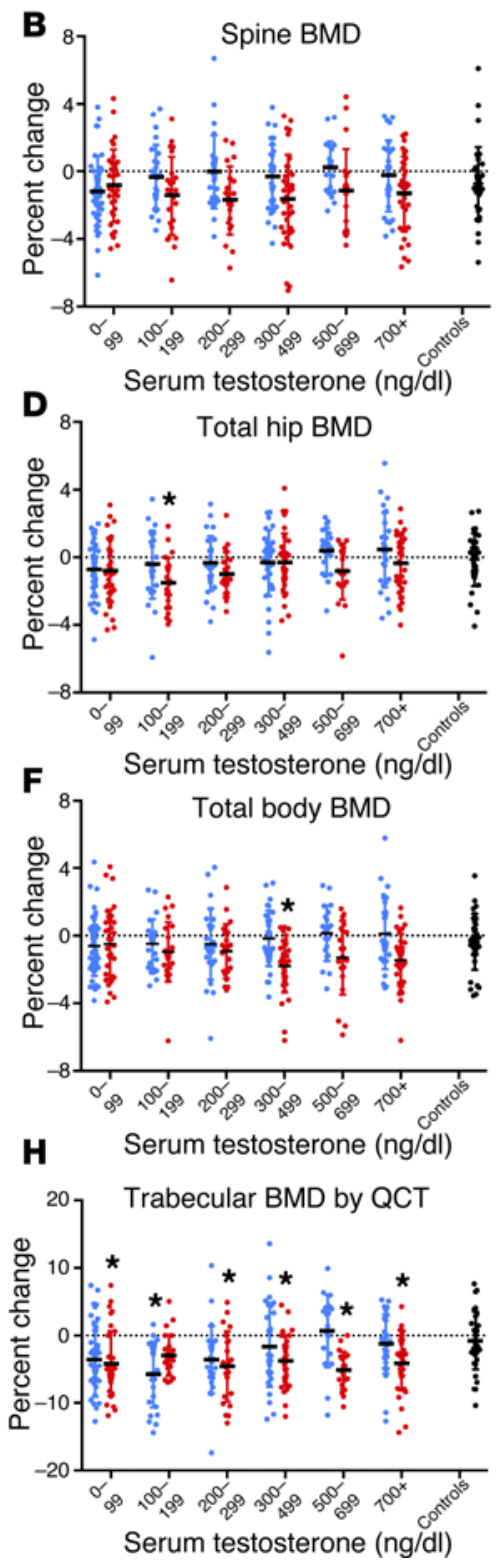

H Serum testosterone (ng/dl)

Figure 4. Percent change from baseline in BMD according to testosterone dose and mean serum testosterone levels on treatment in individual subjects. (A-F) Results are shown for BMD by DXA at the lumbar spine ( $A$ and B), total hip (C and $\mathbf{D})$, and total body (E and $\mathbf{F}$ ). (G and $\mathbf{H}$ ) Spine trabecular BMD by QCT is also shown. Subjects in cohort 1 (blue dots, $n=174$ for $\mathbf{A}-\mathbf{F}$ and $n=169$ for $\mathbf{G}$ and $\mathbf{H}$ ) received goserelin acetate plus 0 (placebo), 1.25, 2.5, 5, or $10 \mathrm{~g}$ of testosterone gel daily without anastrozole. Subjects in cohort 2 (red dots, $n=171$ for $\mathbf{A}-\mathbf{F}$ and $n=168$ for $\mathbf{G}$ and H) received the same treatments plus $1 \mathrm{mg}$ anastrozole per day. Subjects in cohort 3 (black dots, $n=34$ ) received placebos for both goserelin acetate and for the testosterone gel. The horizontal black line represents the mean, and the error bars represent $\pm 1 \mathrm{SD}$. T, testosterone. ${ }^{*} P<0.05$ using Duncan's multiple range test.

Although increases in indices of bone resorption with selective estrogen deficiency likely reflect an independent role of estradiol on bone, it is also possible that estrogen deficiency could alter the metabolism of biochemical indices of bone turnover. Changes in BMD, however, cannot be attributed to theoretical alterations in the clearance of bone turnover markers. Thus, because estradiol deficiency reduced trabecular BMD at the spine, as well as cortical and trabecular VBMD at the distal radius and tibia, the present study provides important new evidence substantiating the role of estradiol deficiency in the pathogenesis of hypogonadal bone loss. When considered together with our prior findings that estrogen deficiency plays a key role in fat accumulation and sexual dysfunction in hypogonadal men (10), it is now clear that estrogen deficiency plays an important, if not dominant, role in many of the key clinical features of male hypogonadism. Classifying features of male hypogonadism based on their relationship to androgen or estrogen deficiency might permit tailoring of therapies for features of male hypogonadism based on their underlying pathogenesis.

Whether androgen deficiency also exerts an independent effect on bone homeostasis in adult men is less clear. While it is feasible to administer testosteror total body, spine BMD by QCT, or biochemical markers of bone turnover (20). In the current study, we extended previously published findings by examining effects of estrogen deficiency over a wide range of testosterone levels. By administering low doses of testosterone without aromatase blockade (as in cohort 1), we were able to determine the minimal levels of gonadal steroids needed to prevent increases in bone resorption, a finding that should have important clinical implications. By administering a wide range of testosterone doses together with a potent aromatase inhibitor (as in cohort 2), we demonstrated that, as long as estradiol levels remain low, bone resorption increases markedly, even if serum testosterone levels are frankly elevated. This finding provides compelling evidence of a powerful and independent effect of estrogen deficiency on bone in men. Additionally, our data demonstrate that estradiol deficiency primarily affects cortical bone, particularly by increasing cortical porosity, with little or no effect on trabecular number or thickness. one to men with low estradiol levels, we did not administer estradiol to testosterone-deficient men because of concerns that some men would develop gynecomastia if exposed to such a hormonal milieu for several months. Thus, we cannot determine if testosterone deficiency by itself contributes to the skeletal effects of male hypogonadism. One group did administer estradiol to testosterone-deficient men, albeit for only 3 weeks to minimize safety concerns, and reported a significant increase in bone resorption indices, though the increase was much smaller than when men were rendered selectively estrogen deficient (17). In the current study, the decline in bone resorption with increasing doses of testosterone in cohort 2 that persisted after adjustment for the small increase in estradiol levels is consistent with an independent effect of androgens on bone resorption, though the skeletal effects of androgens appear to be considerably less potent than the effects of estrogens. 
By serum E levels
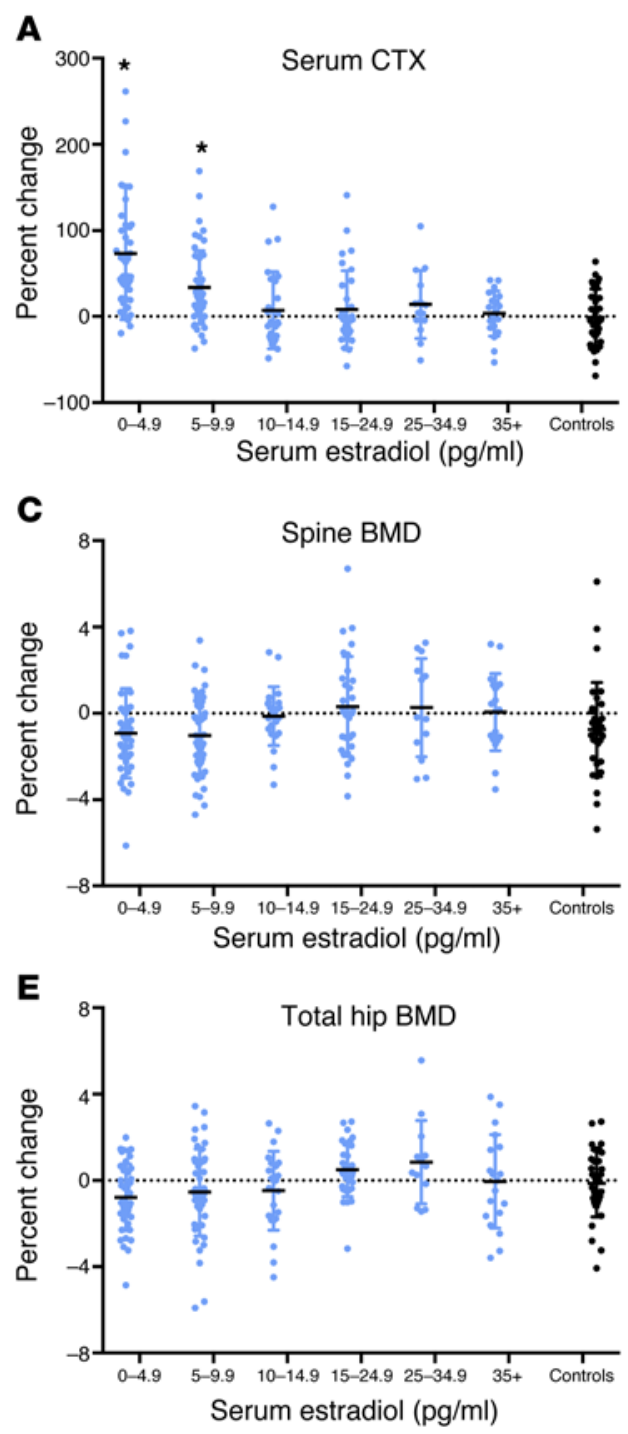

B

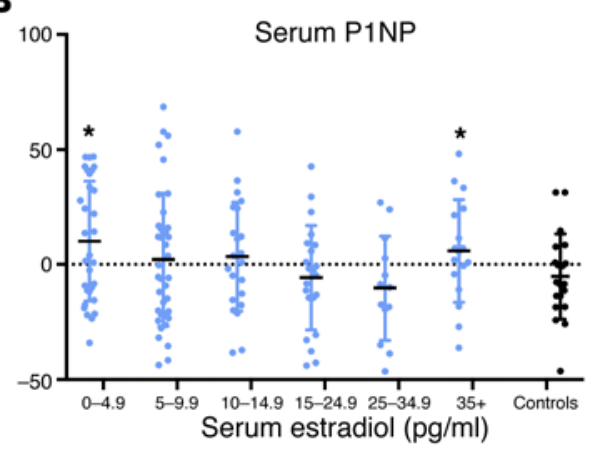

D

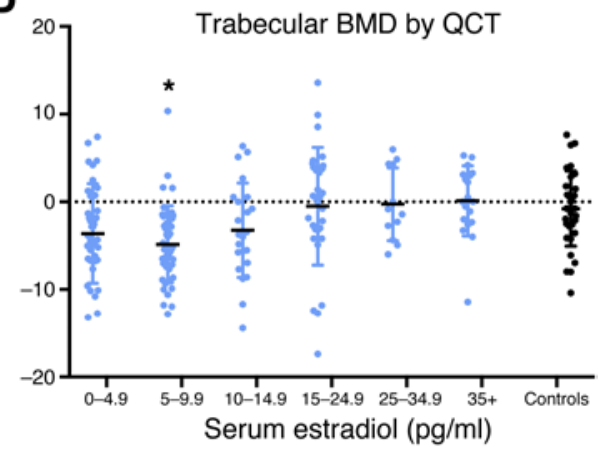

$\mathbf{F}$

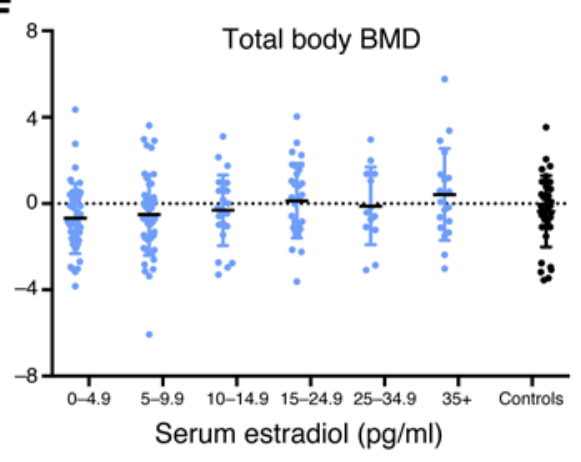

Figure 5. Percent change from baseline in bone turnover markers and BMD according to mean serum estradiol levels on treatment in individual subjects in cohort 1. (A-F) Results are shown for CTX $(n=184$, A), P1NP $(n=184$, B), lumbar spine BMD by DXA $(n=174, \mathrm{C})$ and QCT $(n=171, \mathbf{D})$, total hip BMD by DXA $(n=174, E)$, and total body BMD by DXA $(n=174, F)$. Subjects in cohort 1 (blue dots, $n=198$ ) received goserelin acetate plus 0 (placebo), 1.25, $2.5,5$, or $10 \mathrm{~g}$ of testosterone gel daily without anastrozole. Subjects in cohort 2 had uniformly low estradiol levels due to anastrozole and are therefore not depicted on this graph. Subjects in cohort 3 (controls, black dots, $n=$ 35 for $\mathbf{A}$ and $\mathbf{B}, n=34$ for $\mathbf{C}-\mathbf{F}$ ) received placebos for both goserelin acetate and for the testosterone gel. The horizontal black line represents the mean, and the error bars represent $\pm 1 \mathrm{SD} .{ }^{*} P<0.05$ using Duncan's multiple range test.
The effects of gonadal steroids on bone have also been investigated extensively in animals, though with variable results and conclusions. Bone mass is reduced in male mice with null mutations in either the ER- $\alpha$ gene or the aromatase gene, demonstrating a clear effect of estrogens on bone (21-23). Bone mass is also reduced in male mice with null mutations in the AR gene, indicating that androgens also exert effects on bone (24). Although these genetic mouse models provide convincing evidence that gonadal steroids have powerful effects on bone, these models most likely reflect effects of gonadal steroids on bone development, not effects on adult bone homeostasis. Animal studies examining effects of gonadal steroids on adult bone have reached vastly different conclusions. For example, in male rats, BMD of the femur and lumbar vertebrae declined to a similar extent in animals that underwent orchiectomy or that were treated with a potent aromatase inhibitor, which caused selective estrogen deficiency (25). These data suggest that all of the effects of severe acquired androgen and estrogen deficiency on bone density in male rats can be attributed to the effects of estrogen deficiency alone. However, in mice with low bone mass due to null mutations in ER- $\alpha$, orchiectomy caused a further reduction in bone mass, indicating that even when estrogen action is completely eliminated, androgen withdrawal still exerts an independent effect on bone in male mice (26). Finally, both aromatizable and nonaromatizable androgens can prevent bone loss from orchiectomy in aged male rats, even when combined with an aromatase inhibitor (27-29). This finding suggests that all of the effects of gonadal steroids on bone in male rats can be attributed to the effects of androgens. These conflicting results from animal models are difficult to reconcile, though the use of supraphysiological doses of androgens in some studies may confound the interpretation of the physiological effects of gonadal steroids.

To reconcile these findings from animal and human studies, it has been hypothesized that androgens act primarily on trabecular bone while estrogens act primarily on cortical bone. In male mice with a targeted deletion in the AR gene in osteoblast lineage cells, trabecular bone volume and trabecular number are reduced, demonstrating that androgens exert effects on trabecular bone directly via the $\mathrm{AR}(30,31)$. In contrast, targeted deletion of the ER- $\alpha$ gene in osteoblast lineage cells had no effect on trabecular 
Table 2. Mean ( $\pm S D$ ) percent change from baseline in volumetric BMD and microarchitecture at the distal radius and tibia among men receiving anastrozole and testosterone (cohort 2 ).

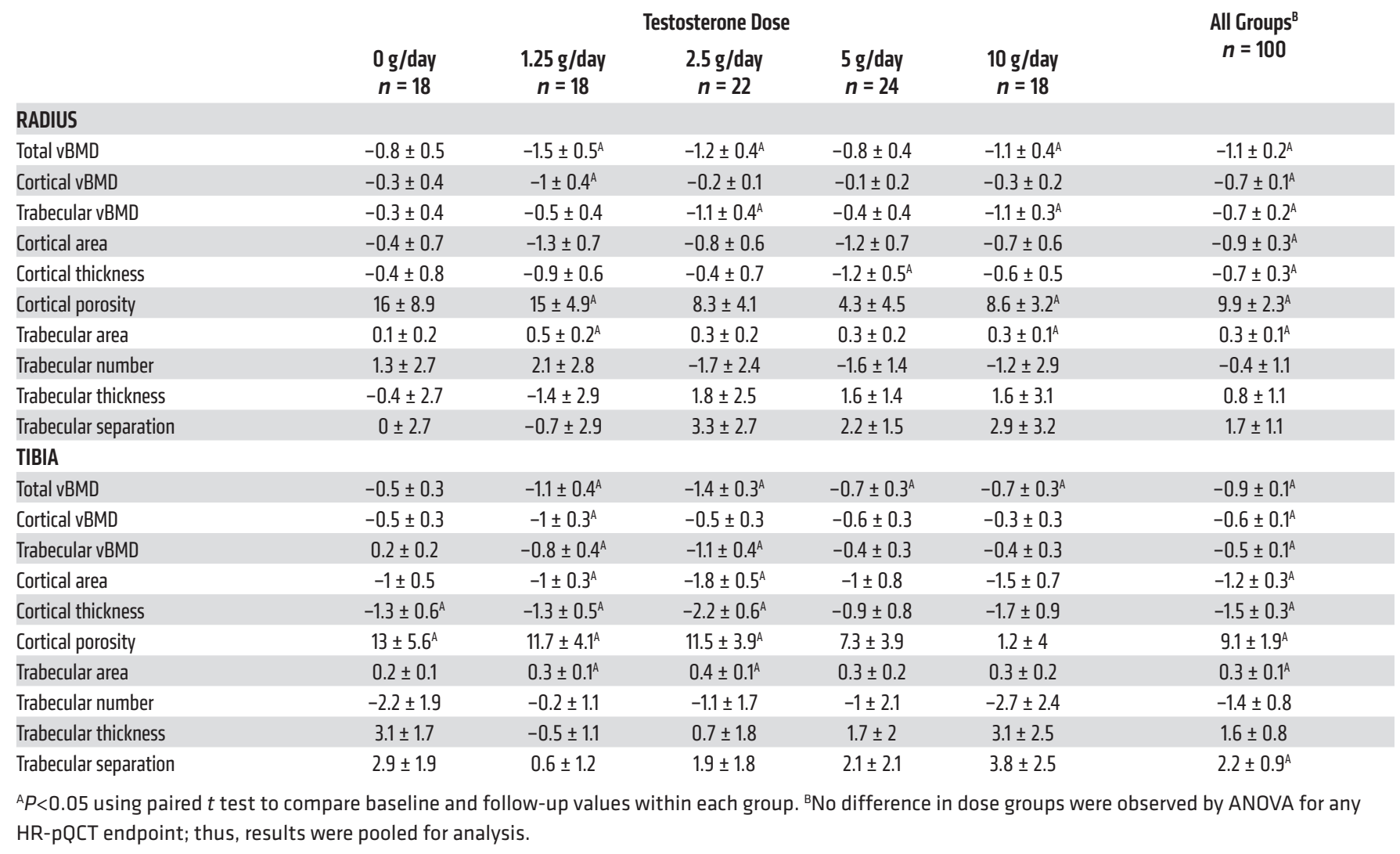

bone in male mice (32). In clinical studies, it has been postulated that the effect of androgens on trabecular bone is difficult to detect utilizing biochemical markers of bone resorption because changes in bone resorption markers reflect the effects of gonadal steroids on the entire skeleton, which is mainly composed of cortical bone (31). Furthermore, our findings from HR-pQCT do suggest that changes in cortical bone were due largely to estrogen deficiency. However, we did not find any evidence of a testosterone effect on trabecular bone, either at the lumbar spine by QCT or at the distal radius or tibia by HR-pQCT. Instead, we found that trabecular BMD loss was also regulated solely by estrogen. Our data are also consistent with cross-sectional studies that have found that serum estradiol is significantly associated with cortical and trabecular bone microarchitecture at peripheral sites in older men $(33,34)$. It remains possible, however, that the regulation of cortical and trabecular bone by gonadal steroids varies throughout the skeleton.

There is no consensus regarding the levels of gonadal steroids at which replacement should begin for skeletal health in hypogonadal men. This difficulty arises, at least in part, because the relationships between gonadal steroid levels and indices of bone health are likely better represented by a continuum rather than a distinct threshold. Nonetheless, clinicians must ultimately decide whether to give hormone replacement to each hypogonadal man. That decision is often based, in large part, on the patient's testosterone and/or estradiol levels, though it should be individualized and other factors should be considered. Although our data suggest that estrogen deficiency plays the dominant pathophysiologic role in hypogonadal bone loss, reliable estradiol assays are not widely available for values in the range seen in hypogonadal men. In those instances, serum testosterone levels appear to serve as a reasonable proxy for estradiol levels. In men who received testosterone without an aromatase inhibitor (cohort 1), our data demonstrate that serum CTX levels increased significantly when serum testosterone levels were below $200 \mathrm{ng} / \mathrm{dl}$ (Figure 3B) or when estradiol levels were below $10 \mathrm{pg} / \mathrm{ml}$ (Figure $5 \mathrm{~A})$. Moreover, trabecular BMD by QCT also appeared to decline in men with whose testosterone and estradiol levels were below these limits (Figure $4 \mathrm{H}$ and Figure 5D). Thus, based on changes in bone resorption markers and trabecular BMD of the lumbar spine, serum estradiol levels below $10 \mathrm{pg} / \mathrm{ml}$ and/or serum testosterone levels below $200 \mathrm{ng} / \mathrm{dl}$ (with intact aromatization) appear to be undesirable for bone health.

Several prior findings also suggest that bone health may be compromised and that a favorable response to testosterone replacement may be more likely when serum testosterone levels are below 200 ng/dl. For example, in the Osteoporotic Fractures in Men Study (MrOS), osteoporosis and rapid bone loss of the proximal femur were more common in older men with serum testosterone levels below $200 \mathrm{ng} / \mathrm{dl}$ (35). In a cohort of men with acquired secondary hypogonadism, whose mean testosterone level was $185 \mathrm{ng} / \mathrm{dl}$, administration of testosterone enanthate increased lumbar spine BMD more than did a placebo (36). Moreover, testosterone administration did 
not increase BMD significantly in men whose mean baseline testosterone level was $367 \mathrm{ng} / \mathrm{dl}$, though a post hoc regression analysis suggested that testosterone administration would clearly have increased BMD if enrollment had been restricted to men whose baseline serum testosterone levels were below $200 \mathrm{ng} / \mathrm{dl}$ (37). In a similar study, however, testosterone administration increased lumbar spine BMD significantly in men whose mean baseline serum testosterone level was approximately $290 \mathrm{ng} / \mathrm{dl}$ (38). In the absence of data demonstrating that testosterone administration reduces fracture risk, and in light of controversial data suggesting that testosterone administration may increase the risk of cardiovascular disease $(39,40)$, current guidelines recommend that the use of testosterone for bone health in hypogonadal men be restricted to men with a moderate to high fracture risk and with serum testosterone levels below $200 \mathrm{ng} / \mathrm{dl}$ (41). Additionally, the decision to treat an individual patient with testosterone should be based on its potential effects on multiple organ systems, not just bone.

Even though the preponderance of data indicate that estradiol is the major regulator of bone metabolism in adult men, very little is known about the level(s) of estradiol needed to ensure stability of the skeleton, and current treatment recommendations do not consider estradiol levels. Observational data have suggested that bone loss accelerates when levels are below $31 \mathrm{pg} / \mathrm{ml}$. In elderly men treated with raloxifene, bone resorption increased if baseline serum estradiol levels were below $26 \mathrm{pg} / \mathrm{ml}$. Based on these findings, older studies proposed that men need estradiol levels of at least $20-30 \mathrm{pg} / \mathrm{ml}$ to prevent bone loss, values that are much higher than our estimates and that are similar to the baseline estradiol values in most of our healthy young men. These discrepancies may be due, at least in part, to the difficulty in measuring low levels of estradiol by radioimmunoassay. If a well-validated estradiol assay using mass spectroscopy is available, then our data suggest that men whose estradiol levels are below $10 \mathrm{pg} / \mathrm{ml}$ are at risk for significant bone loss and that this risk is even greater in men whose estradiol levels are below $5 \mathrm{pg} / \mathrm{ml}$. If mass spectroscopic measurements of estradiol are not available, then measuring serum testosterone by radioimmunoassay may provide a clinically useful surrogate method to assist in deciding whether hormone replacement is warranted.

Some limitations of our study deserve mention. First, these results were obtained in 20- to 50-year-old men. It is unknown if similar results would be obtained in older men. Second, though most circulating estradiol is derived from aromatization of circulating testosterone, a small portion is directly secreted by the testes in normal men and may not be restored with exogenous testosterone administration (42). Third, we studied the effects of acute hypogonadism in men. It is possible that the skeletal effects of long-term hypogonadism may differ. Fourth, our model is not well suited to determine the role of isolated testosterone deficiency on bone. Although the pattern of changes in bone microarchitecture in cohort 2 is most consistent with a dominant effect of estradiol, the absence of measurements of bone microarchitecture in cohort 1 leaves open the possibility that changes from baseline in groups that received low doses of testosterone could be due, at least in part, to androgen deficiency. Finally, as discussed above, it is unlikely that a distinct threshold level of testosterone or estradiol exists below which bone loss occurs and above which bone homeostasis is normal. The relationship between changes in BMD and/or bone turnover versus levels of testosterone or estradiol is likely a continuum, and the levels of gonadal steroids that are protective against bone loss likely vary between individuals and even within the same man, depending on a plethora of other factors that affect bone homeostasis.

In conclusion, these findings demonstrate that the skeletal consequences of hypogonadism in men are primarily due to estrogen deficiency. Although the relationship between high-turnover bone loss and gonadal steroid levels is likely a continuum, the risk of hypogonadal bone loss appears to accelerate when serum estradiol levels fall below $10 \mathrm{pg} / \mathrm{ml}$ and/or serum testosterone levels fall below $200 \mathrm{ng} / \mathrm{dl}$. The recognition that hypogonadal bone loss is primarily due to estrogen deficiency provides additional support for the growing body of evidence supporting the view that the various features of male hypogonadism be classified based on their relationship to androgen or estrogen deficiency. Characterizing the distinct roles of androgens and estrogens in various features of male hypogonadism should facilitate the development of more rational diagnostic and therapeutic approaches to the care of hypogonadal men.

\section{Methods}

Recruitment and randomization of participants. Healthy men 20-50 years old were recruited to this randomized placebo-controlled trial from September 2004 to December 2010 as previously described (10). Briefly, participants were recruited by sending letters to men in the local area who were identified using commercially available mailing lists that provided the subjects' age and sex. A computerized program was used to randomize subjects in cohorts 1 and 2 using permuted blocks. The block sizes were also randomly determined. The controls were recruited separately from cohorts 1 and 2 . However, they were recruited using the same methodologies as for cohorts 1 and 2 . All subjects, including the controls, were blinded as to their treatment assignment, as were the individuals who interpreted all of the bone density scans. All men had screening serum testosterone levels within our local reference range (270-1,070 ng/dl). Men using medications or with disorders known to affect bone metabolism were excluded.

Study design and protocol. Subjects in cohort $1(n=198)$ received goserelin acetate (Zoladex, provided by AstraZeneca Pharmaceuticals LP) $3.6 \mathrm{mg}$ s.c. at weeks $0,4,8$, and 12 to suppress endogenous production of gonadal steroids. Men were then randomized to one of five testosterone replacement groups that received 0 (placebo), 1.25, 2.5, 5 , or 10 grams of a topical 1\% testosterone gel (AndroGel, provided by AbbVie Inc.) daily for 16 weeks. Subjects in cohort $2(n=202)$ were randomized to the same treatments as cohort 1 but also received $1 \mathrm{mg}$ anastrozole (Arimidex, provided by AstraZeneca Pharmaceuticals LP) daily to suppress aromatization of testosterone to estradiol. A computerized program was used to randomly assign participants in permuted blocks. The block sizes were also randomly determined. The controls (cohort 3, $n=37$ ) received placebos for both goserelin acetate and the testosterone gel. Subjects were seen every four weeks. At each visit, fasting blood was collected to measure serum testosterone and estradiol, CTX, P1NP, and routine safety measures (complete blood count, automated chemistry panel, and liver function tests). Medication compliance was assessed by reviewing diaries and with a structured interview. At baseline and week 16, areal BMD of the proximal femur, 
lumbar spine, and total body was measured using DXA (Hologic QDR 4500A), and trabecular BMD of the spine was determined by QCT at the level of the L4 vertebral body using a GE LightSpeed Pro 16 scanner (GE Healthcare) (36). Our short-term in vivo coefficients of variation for posterior-anterior spine and total hip DXA measurements were $0.014 \mathrm{~g} / \mathrm{cm}^{2}(1.4 \%)$ and $0.016 \mathrm{~g} / \mathrm{cm}^{2}(2.2 \%)$, respectively. Within cohort 2, 100 subjects also underwent HR-pQCT scans (XtremeCT, Scanco Medical) as previously described (43) to assess volumetric bone density and microarchitecture at the distal radius and tibia. The remainder of the subjects did not undergo HR-pQCT because they completed the study prior to acquisition of the HR-pQCT machine. The standard analysis program (Scanco Medical software version V6.0) was used to calculate trabecular geometry, vBMD, and microarchitecture. To characterize cortical microarchitecture in greater detail, HR-pQCT images were processed by a semiautomated cortical bone segmentation technique as previously described (44). After image segmentation, measures were obtained for cortical geometry, density, and porosity, as well as for trabecular area and total area. Our shortterm in vivo coefficients of variation for HR-pQCT measurements at the radius and tibia ranged from $0.2 \%-1.4 \%$ for vBMD parameters; $0.3 \%-8.6 \%$ for trabecular microarchitecture parameters; $0.6 \%-2.4 \%$ for cortical microarchitecture parameters; $7.3 \%-20.2 \%$ for cortical porosity measurements; and $2.1 \%-3.0 \%$ for microfinite element analysis (mFEA) measures. Subjects who discontinued study medication after week 8 but before week 16 were permitted to undergo follow-up DXA, QCT, and HR-pQCT scans at their final visit.

Serum measurements. Total testosterone was measured by solidphase chemiluminescent immunoassay using an automated analyzer (Advia Centaur XP, Siemens). The assay sensitivity was $20 \mathrm{ng} / \mathrm{dl}$. Total testosterone was remeasured by liquid chromatography-tandem mass spectroscopy at all time points from 5 randomly selected men in each of the five groups in cohort 1 and the controls (45). The correlation between the testosterone assays was 0.93 and the assays gave very similar results $\left(\mathrm{T}_{\mathrm{RIA}}=0.98 \mathrm{~T}_{\mathrm{LC} / \mathrm{MS} / \mathrm{MS}}+21\right)$. Estradiol was measured using liquid chromatography-tandem mass spectroscopy. The assay sensitivity was $1.25 \mathrm{pg} / \mathrm{ml}$ (45). Serum CTX levels were measured by ELISA (CrossLaps Ò, Immunodiagnostic Systems Limited), and serum P1NP levels were measured by radioimmunoassay (Orion Diagnostica).

Progression of subjects through study protocol. One hundred eightyfour men in cohort 1, 174 men in cohort 2, and 35 controls completed the study without protocol violations at least through week 8 and were therefore included in the analysis of the primary end point, serum CTX. In cohort 1, DXA data were unavailable for 10 men who dropped out before week 8 (and thus were excluded from all analyses), 8 men who dropped out between weeks 8 and 16 (and did not undergo an early termination visit), 4 men with protocol violations, and 2 men because of scheduling difficulties or machine malfunction. QCT data were unavailable for 10 men who dropped out before week 8, 8 men who dropped out between weeks 8 and 16 (and did not undergo an early termination visit), 4 men with protocol violations, and 5 men because of scheduling difficulties or machine malfunction, leaving 174 and 171 men from cohort 1 in the DXA and QCT analyses, respectively. In cohort 2, DXA data were unavailable for 27 men who dropped out before week 8 (and thus were excluded from all analyses), 4 men who dropped out between weeks 8 and 16 (and did not undergo an early termination visit), one man with a protocol violation, and one man because of machine malfunction, while QCT data were unavail- able for 27 men who dropped out before week 8, 4 men who dropped out between weeks 8 and 16 (and did not undergo an early termination visit), one man with a protocol violation, and 2 men because of scheduling difficulties or machine malfunction, leaving 169 and 168 men from cohort 2 in the DXA and QCT analyses, respectively. In the controls, DXA and QCT data were unavailable for 2 men who dropped out before week 8 , and one man who dropped out between weeks 8 and 16 did not undergo an early termination visit, leaving 34 men in the DXA analysis and 34 men in the QCT analysis. Among the subset of men in cohort 2 who received HR-pQCT, radius HR-pQCT scans were unusable in 4 men and tibia HR-pQCT were unsable in 3 men due to motion artifact, leaving 96 and 97 men in the radius and tibia HR-pQCT analyses, respectively.

Statistical analysis and data interpretation. Baseline characteristics of the cohorts were compared using ANOVA to assess intracohort differences between testosterone-dose groups and two-tailed independent $t$ tests to assess intercohort differences for each of the dose groups. The primary outcome variable was the change in serum CTX. The study was designed to have $80 \%$ power at a significance level of $\alpha=0.025$ using one-way ANOVA to detect a difference in 16 -week mean changes of at least $0.3 \times$ its common standard deviation based on a sample size of 40 subjects per group and assuming that $80 \%$ of subjects have usable data. This study was not designed to have sufficient power to detect significant changes in BMD by DXA in cohort 1 . There was insufficient information available to perform power assessments for cohort 2. Because we were assessing changes in outcome variables, only subjects who remained on their assigned therapy until week 8 and missed fewer than $20 \%$ of their study medication doses were included in the data analysis. All statistical tests are 2 -sided. $P$ values less than 0.05 were considered significant. Unless otherwise noted, data are presented as the mean \pm SD.

Effects of testosterone dose and level on bone resorption and bone loss. We compared changes from baseline to the final visit in each of the testosterone-dose groups for each outcome variable in cohort 1 (aromatization intact) and cohort 2 (aromatization suppressed) with the changes in cohort 3 (controls) using Duncan's multiple range test. This test adjusts results for multiple comparisons. As specified in our analysis plan, to provide results in a format more useful to clinicians, similar analyses were performed in which the men in cohorts 1 and 2 were regrouped according to their average testosterone level from week 4-16 (0-99, 100-199, 200-299, 300-499, 500-699, and $\geq 700$ $\mathrm{ng} / \mathrm{dl})$. Changes in outcome measures for each of those groups were then compared with the changes in the control group. Because serum testosterone levels decline markedly between 24 and 48 hours after testosterone gel application (46), hormone values were excluded for visits in which subjects reported that their last dose was at least 48 hours earlier (13 of 1,050 values). Hormone values from the remaining visits from weeks 4-16 were then averaged.

Effects of estradiol level on bone turnover and bone loss. To assess the estradiol levels below which bone resorption increased and BMD decreased, we grouped the men in cohort 1 according to their average estradiol level during weeks 4-16 (0-4.9, 5-9.9, 10-14.9, 15-24.9, $25-34.9$, and $\geq 35 \mathrm{pg} / \mathrm{ml}$ ) and then compared changes in outcome measures for each of those groups with the changes in the control group using Duncan's multiple range test.

Roles of androgens and estrogens in the regulation of bone turnover and BMD. To determine if androgens themselves contributed to the 
regulation of bone resorption, we compared changes in bone outcomes between pairs of groups within cohort 2 using Duncan's Multiple Range test. Because mean serum testosterone levels should increase in a dose-responsive manner in cohort 2 while serum estradiol levels remained markedly suppressed (10), differences in outcome variables between groups in cohort 2 should reflect an independent effect of androgens. However, because there were small increases in serum estradiol levels as testosterone dose and/or levels increased, analyses were adjusted for ambient estradiol levels. To determine if estradiol exhibited an independent effect on each outcome, we utilized general linear model-based tests in which the effects were cohort, testosterone dose, and the cohort-testosterone dose interaction. Effects of estradiol on each outcome were also assessed using two-tailed independent $t$ tests comparing the mean change in each outcome for all groups that received active testosterone $(1.25,2.5,5$, or 10 grams daily) in cohort 1 with the mean change in the corresponding testosterone-dose groups in cohort 2. Because estradiol levels should be lower in cohort 2 than in cohort 1 , despite similar testosterone levels (10), differences in outcomes between testosterone-treated groups in cohorts 1 and 2 indicate an independent effect of estradiol on each outcome measure.

Roles of androgens and estrogens in the regulation of peripheral $v B M D$ and bone microarchitecture. To assess whether testosterone affects vBMD or bone microarchitecture in the absence of estrogen, we examined differences between testosterone-dose groups in cohort 2 using ANOVA. To determine the effects of suppressing estradiol across a range of testosterone levels, we compared changes from baseline to the final visit in each of the testosterone-dose groups in cohort 2 using two-tailed paired $t$ tests. If no differences were detected between groups using ANOVA, a secondary analysis was performed with twotailed paired $t$ tests among pooled groups to evaluate global effects of estrogen suppression on skeletal endpoints.

Study approval. This study was conducted at Massachusetts General Hospital in Boston, Massachusetts, USA, and was approved by the Institutional Review Board of Partners HealthCare. All subjects pro- vided written informed consent. This protocol was registered at ClinicalTrials.gov (NCT00114114).

\section{Author contributions}

JSF contributed study concept and design. JSF, BZL, SAMBB, DWG, CWH, SCH, AL, NP, ABS, APT, MLW, JMY, and EWY acquired the data. HL and EWY contributed statistical analysis. JSF, EWY, BZL, and SAMBB interpreted the data. JSF and EWY drafted of the manuscript. All authors contributed critical review of manuscript.

\section{Acknowledgments}

We wish to thank Brent Jones, Laurence Borges, Kendra Wulczyn, and Christopher Barry for their dedicated administration of the study protocol and assistance with data management; the staff of the Mallinckrodt General Clinical Research Center for their care of the subjects; the staff of the Massachusetts General Hospital Bone Density Center for performing the bone density and body composition measurements; Robert M. Neer and Henry M. Kronenberg for their scientific guidance, and Deborah Fitzgerald for her administrative support. Supported by NIH grants R01 AG 030545, K24 DK0275, and S10 RR02305 (all to J.S. Finkelstein); NIH grants M01 RR01066 and UL1 RR 25758 (to the Massachusetts General Hospital Clinical Research Center); and an investigator-initiated grant from AbbVie Inc.

AndroGel was provided by AbbVie Inc., Zoladex and Arimidex were provided by AstraZeneca Pharmaceuticals LP. Neither Zoladex nor Arimidex are licensed for use in this setting.

Address correspondence to: Joel S. Finkelstein, Endocrine Unit, Thier 1051, Massachusetts General Hospital, 50 Blossom St., Boston, Massachusetts 02114, USA. Phone: 617.726.3296; E-mail: jfinkelstein@partners.org.
1. Bonjour JP, Theintz G, Buchs B, Slosman D, Rizzoli R. Critical years and stages of puberty for spinal and femoral bone mass accumulation during adolescence. J Clin Endocrinol Metab. 1991;73(3):555-563.

2. Finkelstein JS, et al. Bone mineral density changes during the menopause transition in a multi-ethnic cohort of women. JClin Endocrinol Metab. 2008;93(3):861-868.

3. Feldman HA, et al. Age trends in the level of serum testosterone and other hormones in middle-aged men: longitudinal results from the Massachusetts male aging study. JClin Endocrinol Metab. 2002;87(2):589-598.

4. Harman SM, Metter EJ, Tobin JD, Pearson J, Blackman MR. Longitudinal effects of aging on serum total and free testosterone levels in healthy men. Baltimore Longitudinal Study of Aging. J Clin Endocrinol Metab. 2001;86(2):724-731.

5. Greenspan SL, Coates P, Sereika SM, Nelson JB, Trump DL, Resnick NM. Bone loss after initiation of androgen deprivation therapy in patients with prostate cancer. JClin Endocrinol Metab. 2005;90(12):6410-6417.
6. Smith MR, et al. Pamidronate to prevent bone loss during androgen-deprivation therapy for prostate cancer. N Engl JMed. 2001;345(13):948-955.

7. Cawthon PM, et al. Loss of hip BMD in older men: the osteoporotic fractures in men (MrOS) study. J Bone Miner Res. 2009;24(10):1728-1735.

8. Longcope C, Kato T, Horton R. Conversion of blood androgens to estrogens in normal adult men and women. JClin Invest. 1969;48(12):2191-2201.

9. Khosla S, et al. Relationship of serum sex steroid levels and bone turnover markers with bone mineral density in men and women: a key role for bioavailable estrogen. J Clin Endocrinol Metab. 1998;83(7):2266-2274.

10. Finkelstein JS, Yu EW, Burnett-Bowie SA. Gonadal steroids and body composition, strength, and sexual function in men. $\mathrm{NEnglJ}$ Med. 2013;369(25):2457.

11. Khosla S, Melton LJ, Riggs BL. Clinical review 144: Estrogen and the male skeleton. JClin Endocrinol Metab. 2002;87(4):1443-1450.

12. Amin S, et al. Association of hypogonadism and estradiol levels with bone mineral density in elderly men from the Framingham study. Ann Intern Med. 2000;133(12):951-963.
13. Smith EP, et al. Estrogen resistance caused by a mutation in the estrogen-receptor gene in a man. NEngl JMed. 1994;331(16):1056-1061.

14. Marcus R, Leary D, Schneider DL, Shane E, Favus M, Quigley CA. The contribution of testosterone to skeletal development and maintenance: lessons from the androgen insensitivity syndrome. JClin Endocrinol Metab. 2000;85(3):1032-1037.

15. Carani C, et al. Effect of testosterone and estradiol in a man with aromatase deficiency. $N$ EnglJ Med.1997;337(2):91-95.

16. Morishima A, Grumbach MM, Simpson ER, Fisher C, Qin K. Aromatase deficiency in male and female siblings caused by a novel mutation and the physiological role of estrogens. J Clin Endocrinol Metab. 1995;80(12):3689-3698.

17. Falahati-Nini A, Riggs BL, Atkinson EJ, O'Fallon WM, Eastell R, Khosla S. Relative contributions of testosterone and estrogen in regulating bone resorption and formation in normal elderly men. J Clin Invest. 2000;106(12):1553-1560.

18. Leder BZ, LeBlanc KM, Schoenfeld DA, Eastell R, Finkelstein JS. Differential effects of androgens and estrogens on bone turnover in normal men. $J$ Clin Endocrinol Metab. 2003;88(1):204-210. 
19. Lee H, Finkelstein JS, Miller M, Comeaux SJ, Cohen RI, Leder BZ. Effects of selective testosterone and estradiol withdrawal on skeletal sensitivity to parathyroid hormone in men. JClin Endocrinol Metab. 2006;91(3):1069-1075.

20. Burnett-Bowie SA, McKay EA, Lee H, Leder BZ. Effects of aromatase inhibition on bone mineral density and bone turnover in older men with low testosterone levels. JClin Endocrinol Metab. 2009;94(12):4785-4792.

21. Korach KS. Insights from the study of animals lacking functional estrogen receptor. Science. 1994;266(5190):1524-1527.

22. Oz OK, et al. Bone has a sexually dimorphic response to aromatase deficiency. J Bone Miner Res. 2000;15(3):507-514.

23. Vanderschueren $D$, et al. Sex steroid actions in male bone. Endocr Rev. 2014;35(6):906-960.

24. Vandenput L, et al. Role of the androgen receptor in skeletal homeostasis: the androgen-resistant testicular feminized male mouse model. J Bone Miner Res. 2004;19(9):1462-1470.

25. Vanderschueren D, van Herck E, Nijs J, Ederveen AG, De Coster R, Bouillon R. Aromatase inhibition impairs skeletal modeling and decreases bone mineral density in growing male rats. Endocrinology. 1997;138(6):2301-2307.

26. Vandenput L, et al. Testosterone prevents orchidectomy-induced bone loss in estrogen receptor-alpha knockout mice. Biochem Biophys Res Commun. 2001;285(1):70-76.

27. Venken $\mathrm{K}$, et al. Relative impact of androgen and estrogen receptor activation in the effects of androgens on trabecular and cortical bone in growing male mice: a study in the androgen receptor knockout mouse model. J Bone Miner Res. 2006;21(4):576-585.

28. McCoy SC, et al. 17ß-Hydroxyestra-4,9,11-trien-3one (Trenbolone) preserves bone mineral density in skeletally mature orchiectomized rats without prostate enlargement. Bone. 2012;51(4):667-673.

29. Beck DT, et al. Influence of aromatase inhibition on the bone-protective effects of testosterone. J Bone Miner Res. 2014;29(11):2405-2413.

30. Notini AJ, et al. Osteoblast deletion of exon 3 of the androgen receptor gene results in trabecular bone loss in adult male mice. J Bone Miner Res. 2007;22(3):347-356.

31. Khosla S. New insights into androgen and estrogen receptor regulation of the male skeleton. JBone Miner Res. 2015;30(7):1134-1137.

32. Ucer $\mathrm{S}$, et al. The effects of androgens on murine cortical bone do not require ar or ER $\alpha$ signaling in osteoblasts and osteoclasts. J Bone Miner Res. 2015;30(7):1138-1149.

33. Vandenput L, et al. Serum estradiol levels are inversely associated with cortical porosity in older men. J Clin Endocrinol Metab. 2014;99(7):E1322-E1326.

34. Khosla S, Melton LJ 3rd, Achenbach SJ, Oberg AL, Riggs BL. Hormonal and biochemical determinants of trabecular microstructure at the ultradistal radius in women and men. J Clin Endocrinol Metab. 2006;91(3):885-891.

35. Fink HA, et al. Association of testosterone and estradiol deficiency with osteoporosis and rapid bone loss in older men. J Clin Endocrinol Metab. 2006;91(10):3908-3915.

36. Katznelson L, Finkelstein JS, Schoenfeld DA, Rosenthal DI, Anderson EJ, Klibanski A. Increase in bone density and lean body mass during testosterone administration in men with acquired hypogonadism. JClin Endocrinol Metab. 1996;81(12):4358-4365.

37. Snyder PJ, et al. Effect of testosterone treatment on bone mineral density in men over 65 years of age. $J$ Clin Endocrinol Metab. 1999;84(6):1966-1972. 38. Amory JK, et al. Exogenous testosterone or testosterone with finasteride increases bone mineral density in older men with low serum testosterone. JClin Endocrinol Metab. 2004;89(2):503-510.

39. Finkle WD, et al. and Hoover RN. Increased risk of non-fatal myocardial infarction following testosterone therapy prescription in men. PLoS One. 2014;9(1):e85805.

40. Vigen R, et al. Association of testosterone therapy with mortality, myocardial infarction, and stroke in men with low testosterone levels. JAMA. 2013;310(17):1829-1836.

41. Watts MB, et al. Osteoporosis in men: An Endocrine Society clinical practice guideline. JClin Endocrinol Metab. 2012;97(6):1802-1822.

42. MacDonald PC, Madden JD, Brenner PF, Wilson JD, Siiteri PK. Origin of estrogen in normal men and in women with testicular feminization. JClin Endocrinol Metab. 1979;49(6):905-916.

43. Yu EW, Putman MS, Derrico N, Abrishamanian-Garcia G, Finkelstein JS, Bouxsein ML. Defects in cortical microarchitecture among African-American women with type 2 diabetes. Osteoporos Int. 2015;26(2):673-679.

44. Burghardt AJ, Buie HR, Laib A, Majumdar S, Boyd SK. Reproducibility of direct quantitative measures of cortical bone microarchitecture of the distal radius and tibia by HR-pQCT. Bone. 2010;47(3):519-528.

45. Khosla S, et al. Comparison of sex steroid measurements in men by immunoassay versus mass spectroscopy and relationships with cortical and trabecular volumetric bone mineral density. Osteoporos Int. 2008;19(10):1465-1471.

46. Wang C, et al. Pharmacokinetics of transdermal testosterone gel in hypogonadal men: application of gel at one site versus four sites: a General Clinical Research Center Study. JClin Endocrinol Metab. 2000;85(3):964-969. 